\title{
Searches for Massive Graviton Resonances at the LHC
}

\author{
T. V. Obikhod $(D)$ and I. A. Petrenko \\ Institute for Nuclear Research, National Academy of Sciences of Ukraine, 47 Prosp. Nauki, Kiev 03028, Ukraine \\ Correspondence should be addressed to T. V. Obikhod; obikhod@kinr.kiev.ua
}

Received 17 January 2018; Accepted 21 February 2018; Published 30 April 2018

Academic Editor: Marek Nowakowski

Copyright (C) 2018 T. V. Obikhod and I. A. Petrenko. This is an open access article distributed under the Creative Commons Attribution License, which permits unrestricted use, distribution, and reproduction in any medium, provided the original work is properly cited. The publication of this article was funded by SCOAP ${ }^{3}$.

\begin{abstract}
The Standard Model problems lead to the new theories of extra dimensions: Randall-Sundrum model, Arkani-HamedDimopoulos-Dvali model, and $\mathrm{TeV}^{-1}$ model. In the framework of these models, with the help of computer program Pythia8.2, the production cross sections for Kaluza-Klein particles at various energies at the LHC were calculated. The generation of monojet events from scalar graviton emission was considered for number of extra dimensions $(n=2,4$, and 6) for the energy at the LHC $14 \mathrm{TeV}$. The graviton production processes through the gluon-gluon, quark-gluon, and quark-quark fusion processes are also studied and some periodicity was found in the behavior of the graviton mass spectrum. Production cross sections multiplied by branching fractions were calculated for the massive graviton, $\mathrm{G}$, within Randall-Sundrum scenario and the most probable processes of graviton decay at $13 \mathrm{TeV}, 14 \mathrm{TeV}$, and $100 \mathrm{TeV}$ were counted.
\end{abstract}

\section{Introduction}

The problems with theoretical explanation of vacuum energy as well as dark energy, dark matter, and cosmological constant problems are only the tip of the iceberg of problems in the modern theoretical physics. Some of them are

(i) ordinary matter accounting for about $5 \%$ of mass energy in the Universe and no dark matter candidate in the Standard Model (SM),

(ii) hierarchy problem,

(iii) fine tuning of SM Higgs mass,

(iv) no explanation for fermion masses and mixings and three family structures,

(v) unification of strong, electroweak, and gravitational forces,

(vi) compositeness of leptons and quarks,

It is an experimental fact that there is something we cannot explain within the SM.

As is known, vacuum is produced in the processes of phase transitions in Early Universe and the space-time structure of the physical vacuum exhibits the connection to the structure formation in our Universe. So, the understanding of
Universe formation is deeply connected with the conception of the space-time. According to hierarchy formula [1], Plank energy can be reduced to the energy of about $10 \mathrm{TeV}$ that is achieved at the LHC. So, the phenomena of the Universe formation at the early stages and the accompanying processes of particle physics could be studied at modern colliders. In spite of the fact that no new physics beyond the SM is discovered at the LHC at $13 \mathrm{TeV}$, the planned upgrading of the LHC to high luminosities and energies up to $100 \mathrm{TeV}$ gives the possibility for the discovery of new physics. Among such searches for new physics, the most popular are the experimental searches for the Kaluza-Klein (KK) particles.

Historically, KK theory appeared as the unification of gravitational and electromagnetic interactions due to the proposition of a fifth dimension in addition to the usual four-dimensional space-time [2-4], which leads to the consideration of the concept of deformation of Riemannian geometry defined by extrinsic curvature of the space-time. The conclusions of this result are based, in particular, on the five-dimensional space from the paper [5]. Arkani-Hamed et al. proposed the solution to the hierarchy problem on the basis of the existence of new compact spatial dimensions. KK excitations in this extra dimensional space through the combined effect of all the gravitons became observable. 


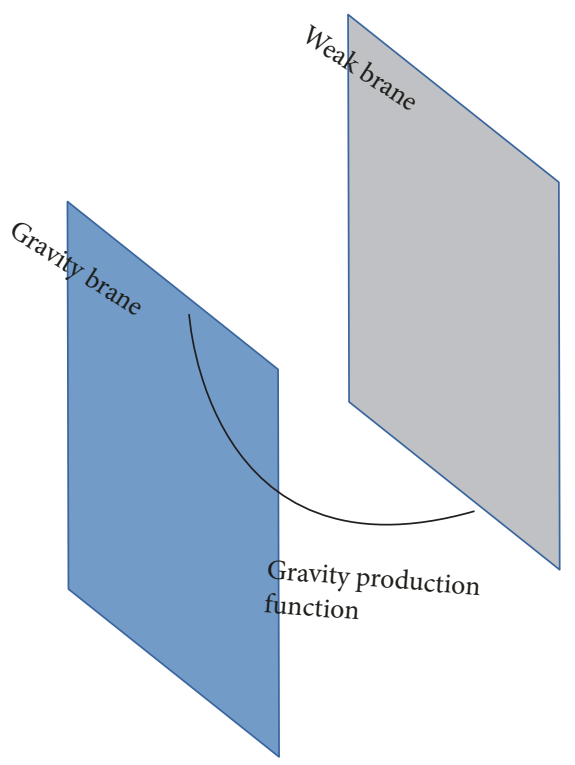

FIGURE 1: RS theory presented by the gravity and weak branes as the 4-dimensional boundaries of the extra dimension (from [12]).

Today, the idea of additional space as the instrumentation of the unification of all four interactions is of interest not only in theoretical physics [6-8] but also in experimental searches at the LHC for exotic matter that deviates from normal matter [9].

Our paper is devoted to the searches for KK particles in three models of extra dimensions: Arkani-HamedDimopoulos-Dvali (ADD) model, [6], Randall-Sundrum (RS) model [7, 8], and $\mathrm{TeV}^{-1}$ model [10]. Using computer program Pythia8.2 [11], within these three extra dimensional models, we have calculated

(i) the production cross section of KK modes of massive gravitons and gauge bosons at energies from $14 \mathrm{TeV}$ to planned $100 \mathrm{TeV}$,

(ii) the graviton mass spectrum for three graviton, $G$, emission processes: (a) gg $\rightarrow G g$, (b) qg $\rightarrow G q$, and (c) $q \bar{q} \rightarrow G g$ at $14 \mathrm{TeV}$ at the LHC,

(iii) the graviton mass spectrum at $14 \mathrm{TeV}$ at the $\mathrm{LHC}$ for numbers of extra dimensions ( $n=2,4$, and 6) (for simplicity and brevity). Since the maximum of $n$ is equal to 6 for ADD model, it was of interest to look at the behavior of graviton mass spectrum at the extreme values of $n$, from $n=2$ to $n=6$,

(iv) the production cross section of graviton, $g g \rightarrow G$, multiplied by branching ratios, $\operatorname{Br}(G \rightarrow g g)$ (gluongluon (gg) pair), $\operatorname{Br}(G \rightarrow l l)$ (leptons, $l$ are of any type, $e, \mu, \tau)$, and $\operatorname{Br}(G \rightarrow h h)$ (h, Higgs boson) of the most probable processes of decay within RS model at $13 \mathrm{TeV}, 14 \mathrm{TeV}$, and $100 \mathrm{TeV}$.

\section{Models of Extra Dimensions}

In this section, we will observe three models of extra dimensions, $\mathrm{ADD}, \mathrm{RS}$, and $\mathrm{TeV}^{-1}$, which are the base for our further calculations of KK particle properties. In the framework of M-theory [13], the metric of ADD model is as follows:

$$
G_{M N}(x, y)=\eta_{M N}+\frac{2}{M^{1+n / 2}} h_{M N}(x, y),
$$

where $G_{M N}(x, y)$ is the metric of $(4+n)$-dimensional spacetime with compact extra dimensions, where the gravitational field propagates and the SM localized on a 3-brane embedded into the $(4+n)$-dimensional space-time, $\eta_{M N}$ is $(4+n)$ dimensional Minkowski background and $h_{M N}(x, y)$ is the deviation of Minkowski metrics, $M$ is the fundamental mass scale, and $n$ is the number of extra dimensions. Masses of KK modes for ADD model are given by

$$
m_{n}=\frac{1}{R} \sqrt{n_{1}^{2}+n_{2}^{2}+\cdots+n_{d}^{2}}=\frac{|n|}{R} .
$$

Five-dimensional metric of RS model with one extra dimension compactified to the orbifold, $S^{1} / Z_{2}$, is with nonfactorizable geometry:

$$
d s^{2}=e^{-2 \sigma(y)} \eta_{\mu \nu} d x^{\mu} d x^{\nu}+d y^{2} .
$$

Two 3-branes are located at points $y=0$ and $y=\pi R$ of the orbifold with radius, $R$, of $S^{1} . x^{\mu}, \mu=0,1,2,3$, and $\eta_{\mu \nu}$ are four-dimensional coordinates and Minkowski metrics; the function $\sigma(y)$ inside the interval $-\pi R<y<\pi R$ is equal to $\sigma(y)=k|y|$. $(k>0$, dimensional parameter). In Figure 1 a nonfactorizable geometry with one spatial extra dimension is presented as a line segment between two four-dimensional branes, known as Planck and $\mathrm{TeV}$ brane.

Masses of KK particles for RS model are given by

$$
m_{n}=\beta_{n} k e^{-\pi k R},
$$

where $\beta_{n}=3.83,7.02,10.17,13.32, \ldots$ for $n=1,2,3,4, \ldots$. The metric of $\mathrm{TeV}^{-1}$ model for ten-dimensional string theory 


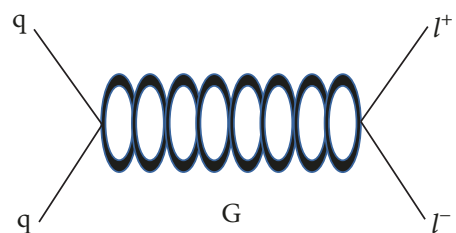

(a)

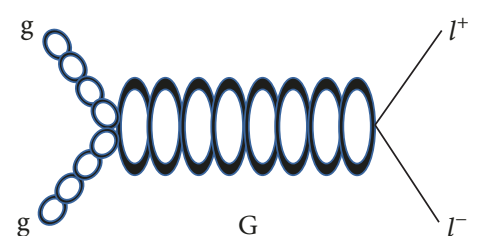

(b)

FIGURE 2: Processes for the graviton resonance production through (a) quark-quark and (b) gluon-gluon fusion.

is determined by the conditions on Calabi-Yau manifold: Ricci-flatness of metric, vanishing first Chern class, and $\mathrm{SU}(n)$ holonomy. Low-energy effective action has much smaller scale than Planck mass related to an internal compactification radius. In these scenarios, the SM fields as well as $\mathrm{Z}_{\mathrm{KK}}$ and $\mathrm{W}_{\mathrm{KK}}$ resonances are allowed to propagate in the bulk, but gravity is not included in the model. Masses of KK modes for $\mathrm{TeV}^{-1}$ model are given by

$$
m_{n}=\left(m_{0}^{2}+\frac{n \cdot n}{R_{c}^{2}}\right)^{1 / 2},
$$

where $n \cdot n$ is scalar production of $n=\left(n_{1}, n_{2}, \ldots\right)$ which labels KK excitation levels, and $m_{0}$ is the mass of gauge zero-mode, which corresponds to SM gauge field.

The advantage of the presented models of extra dimensions lies in the possibility of the observation of the physics of Planck scales, $M_{\mathrm{Pl}}$, at the energies achievable at modern colliders, $M$, due to the presence of extra dimensions, $n$. This result is possible due to the hierarchy formulas for the presented models:

$$
\begin{aligned}
& \text { ADD model: } M_{\mathrm{Pl}}^{2} \sim R^{n} M^{2+n} \\
& \text { RS model: } M_{\mathrm{Pl}}^{2}=\left(M^{3} / k\right)\left(e^{2 k \pi R}-1\right) \\
& \mathrm{TeV}-1 \text { model: hierarchy formula is determ } \\
& \text { low-energy effective action } \\
& \text { 3. Results of Computer Modeling for } \\
& \text { Properties of KK Particles }
\end{aligned}
$$

\section{Results of Computer Modeling for Properties of KK Particles}$$
\mathrm{TeV}^{-1} \text { model: hierarchy formula is determined by the }
$$

RS resonances, connected with production of KK graviton, $\mathrm{G}$, are described in [14]. Experimental searches for massive Graviton production are carried out at the LHC by ATLAS and CMS Collaborations and the latest data on the lower limit on graviton mass are presented in [15]. The production of narrow graviton resonances in the $\mathrm{TeV}$ range at the $\mathrm{LHC}$ as well as the decays into fermion and boson pairs was studied in this paper. For the discovering of graviton resonance, $G$, the parton showering formalism was used, which agrees with the NLO matrix element calculations. The partonic subprocesses are demonstrated in Figure 2.

The ADD graviton emission and virtual graviton exchange processes are described in [16]. Within model with large extra dimensions (LED), the processes that could give rise to new phenomena at LHC due to emission or exchange of gravitons were considered. The implementation of these processes in the Monte Carlo generator Pythia8.2 was presented in this paper. The considered processes are connected with monojet, diphoton, and dilepton final states. It is also possible to generate monojet events from scalar graviton emission as described in [17].

$\mathrm{TeV}^{-1}$ sized extra dimensional KK production processes involve the production of electroweak KK gauge bosons, $\mathrm{Z}_{\mathrm{KK}}$ and $\mathrm{W}_{\mathrm{KK}}$, in one $\mathrm{TeV}^{-1}$ sized extra dimension. The processes are described in $[18,19]$ and allow the specification of final states. In this article, the observation of a certain KK hard process in $\mathrm{pp}$ interactions at the LHC was presented within the $S^{1} / Z_{2}, \mathrm{TeV}^{-1}$ extra dimensional theoretical framework. The analytic form for the hard process cross section has been calculated and has been implemented within the Pythia8.2 Monte Carlo generator.

3.1. The Production Cross Section of KK Particles at Different Energies. With the help of computer program Pythia8.2 for three models of extra dimensions, we have calculated the production cross sections of $\mathrm{KK}$ particles at energies varying from 14 to $100 \mathrm{TeV}$. According to the latest experimental data presented in [15], we reconstructed jets with the anti$\mathrm{k}_{t}$ clustering algorithm, transverse momenta, $p_{T}^{\text {jet }}>20 \mathrm{GeV}$, and rapidity $|\eta| \prec 2.4$ of jets to drop data connected with missing transverse momentum. Events were produced with Monte Carlo event sample using the NNPDF23LO parton distribution function (PDF) for the proton beam and scales of renormalizations and factorizations. The results are presented in Figure 3.

From Figure 3(a), we can see the significant predominance of production for dijet final states above monojet final states within LED model. Figure 3(b) shows that RS KK particles are produced at much higher values than $Z_{\mathrm{KK}}$ and $\mathrm{W}_{\mathrm{KK}}$ bosons in $\mathrm{TeV}^{-1}$ extra dimensional model.

Within $\mathrm{TeV}^{-1}$ model with parameters $n=10$ and $m^{*}=2-10 \mathrm{TeV}$, we calculated the production cross sections at $20 \mathrm{TeV}, 60 \mathrm{TeV}$, and $100 \mathrm{TeV}$ at the center of mass energies as a function of $\mathrm{KK}$ mass, $\mathrm{M}_{\mathrm{Z}_{\mathrm{KK}}}$, presented in Figure 4 . As the decay of $Z_{\mathrm{KK}}$ to muon pair is the dominant one, we decided to calculate, namely, this process of KK particle decay.

From Figure 4, we can see the sharp drop in the curve for $20 \mathrm{TeV}$ compared with other curves at $60 \mathrm{TeV}$ and $100 \mathrm{TeV}$ at the center of mass energies. This result emphasizes the most important result for the further searches of new physics at high energies. As the last two curves $(60 \mathrm{TeV}$ and $100 \mathrm{TeV}$ ) are almost parallel, it is preferable to search for new 

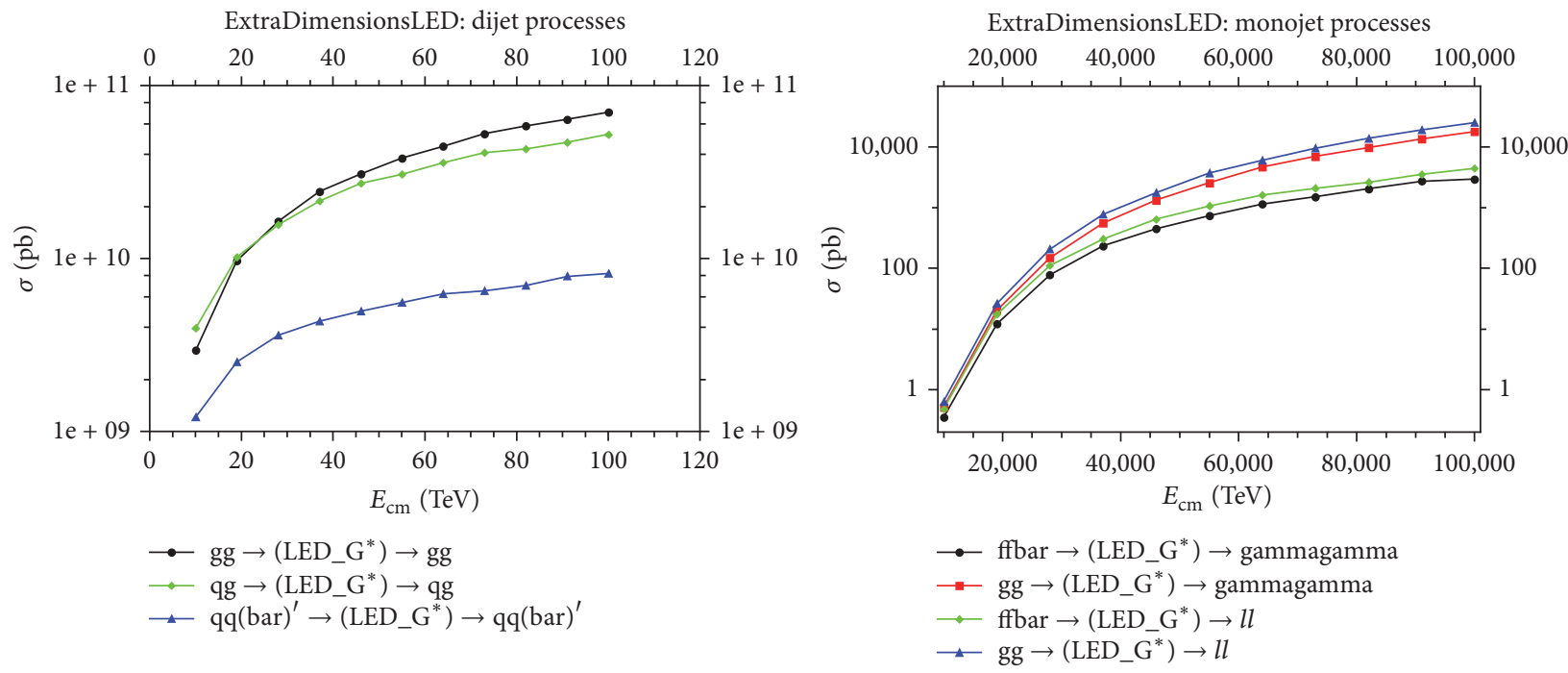

(a)
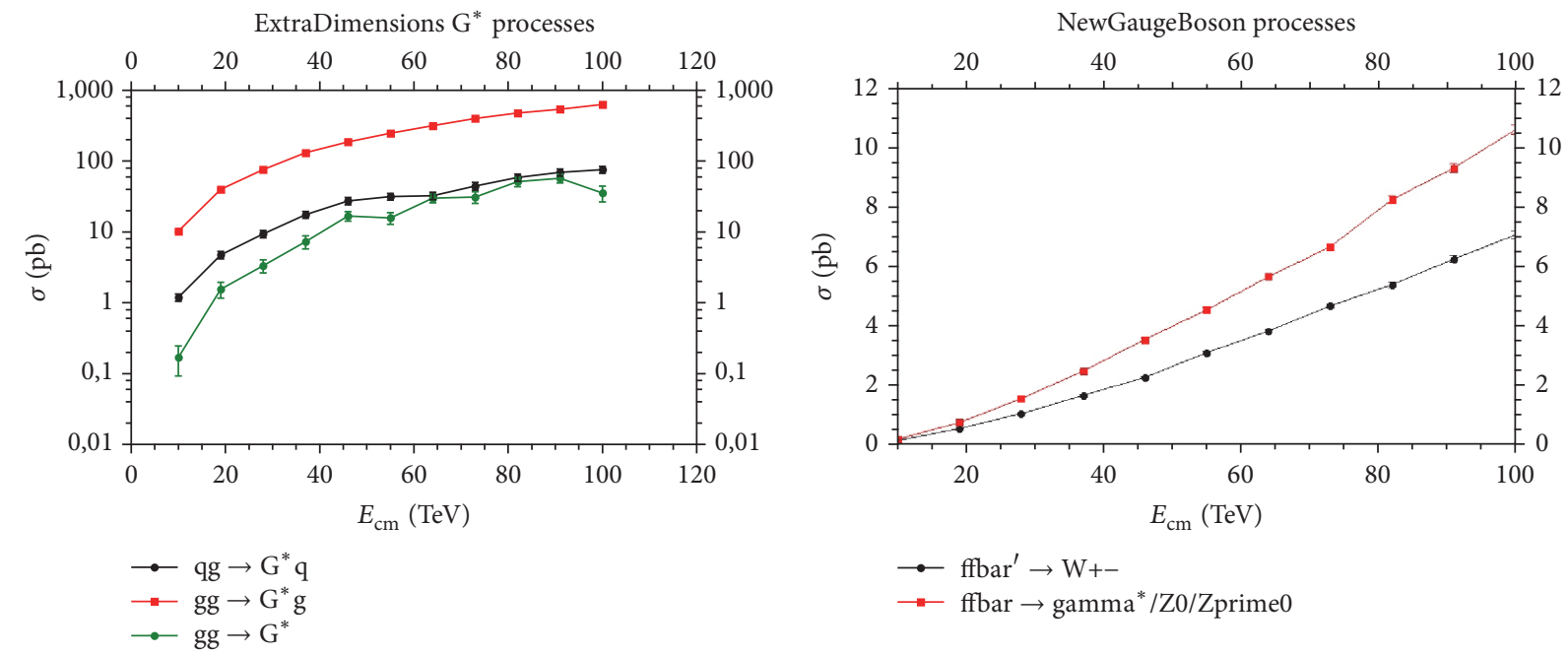

(b)

FIGURE 3: The production cross section at the center of mass energies varying from 14 to $100 \mathrm{TeV}$ for (a) LED model: left panel, dijet final state; right panel, monojet final state; (b) left panel, $\mathrm{RS}$ model; right panel, $\mathrm{TeV}^{-1}$ model.

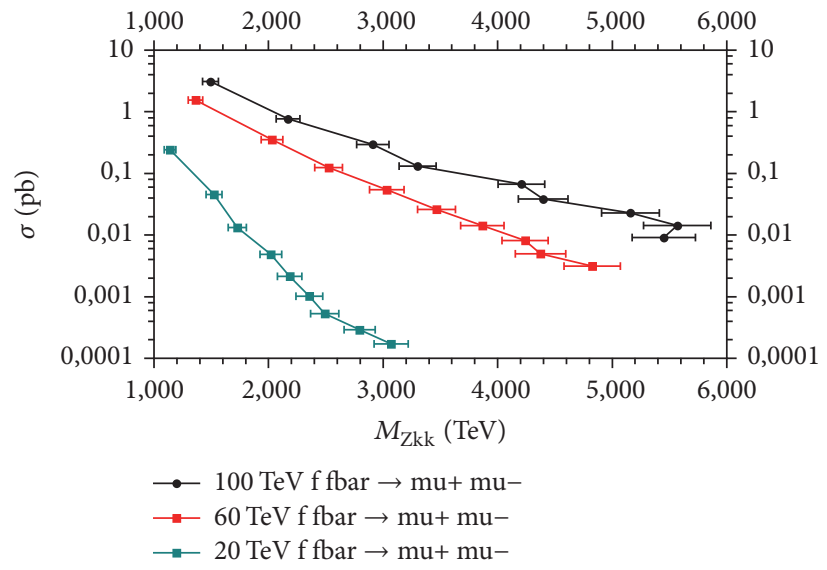

Figure 4: Production cross sections at $20 \mathrm{TeV}, 60 \mathrm{TeV}$, and $100 \mathrm{TeV}$ at the center of mass energies as a function of $\mathrm{KK}_{\text {mass for TeV }}^{-1}$ model. 


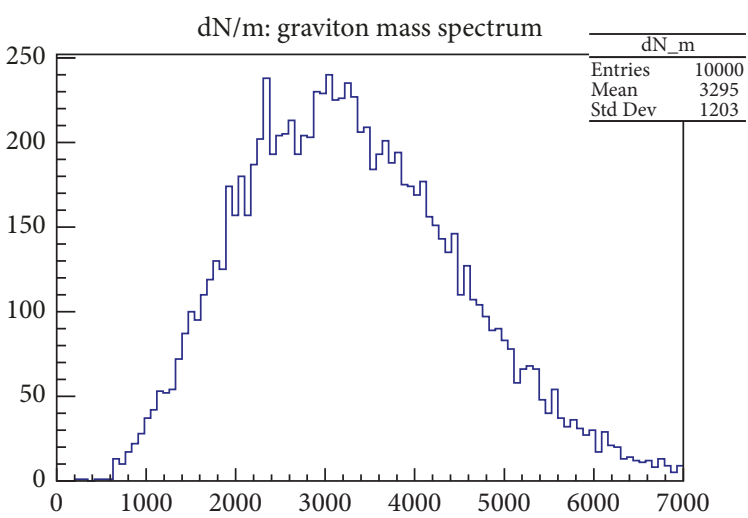

(a)

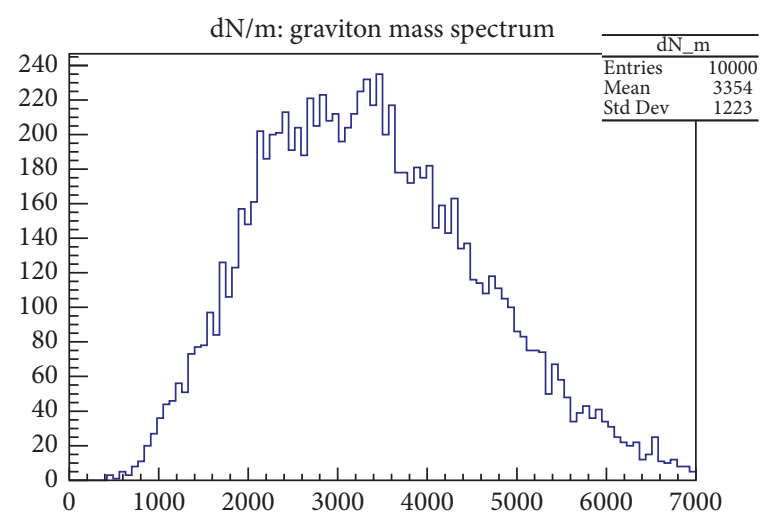

(b)

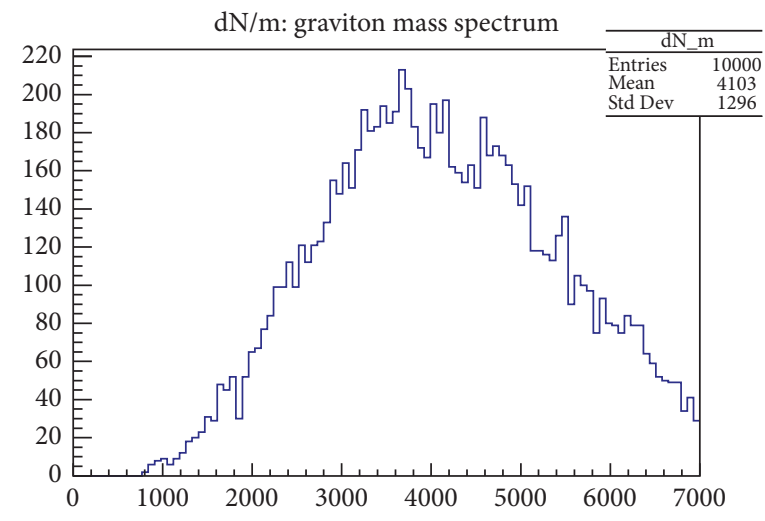

(c)

Figure 5: Graviton mass spectrum within monojet LED model for three graviton, G, emission processes: (a) gg $\rightarrow G g$, (b) qg $\rightarrow G q$, and (c) $q \bar{q} \rightarrow G g$ at the center of mass energies of $14 \mathrm{TeV}$.

phenomena at energies up to $30 \mathrm{TeV}$, when the production cross sections of KK particles can be varied in the wide range.

3.2. The Graviton Mass Spectrum at $14 \mathrm{TeV}$ at the LHC. We will apply large-extra-dimensional (ADD-type) models in production processes for virtual extra dimensional scalars of graviscalar type. With the help of Pythia8.2, it is possible to generate monojet events from scalar graviton emission as described in [17].

The group of lowest-order $\mathrm{G}$ jet emission processes within monojet model was considered with the following parameters: ExtraDimensionsLED: $n=6$; ExtraDimensionsLED: $M$ $=10000$. Three graviton, G, mass spectra for $\mathrm{G}$ jet emission processes, gg $\rightarrow \mathrm{Gg}$ (gluon-gluon fusion (gg) with emission of graviton, G, and gluon, g), qg $\rightarrow G q$ (quark-gluon fusion (qg) with emission of graviton, G, and quark, q), and $q \bar{q} \rightarrow$ $\mathrm{Gg}$ (quark-antiquark fusion $(q \bar{q})$ with emission of graviton, $\mathrm{G}$, and gluon, $\mathrm{g}$ ) at the center of mass energies of $14 \mathrm{TeV}$ at the LHC, are presented in Figure 5.

From Figure 5, the peak of the graviton mass spectrum distribution is viewed depending on the process of monojet emission. Although this dependence is insignificant, nevertheless, for the process $q \bar{q} \rightarrow G g$ of monojet graviton, $G$, emission, it is shifted by almost $1 \mathrm{TeV}$.
As LED model depends on the number of extra dimensions, $n$, it was important to study the graviton mass spectrum distributions for $n=2,4$, and 6 . The results of our calculations of $\mathrm{G}$ jet emission process, $\mathrm{gg} \rightarrow \mathrm{Gg}$, at the center of mass energies of $14 \mathrm{TeV}$ is presented in Figure 6.

From Figure 6, substantial dependence of $\mathrm{G}$ jet emission process in the LED model on the number of extra dimensions is seen. Moreover, we can see clear periodicity of dependence, when peaks are shifted by $1 \mathrm{TeV}$ with an increase of the number of extra dimensions by 2 . According to the hierarchy formula of ADD model, this shifting is connected with the change in the radius of compactification, $R$. As $n$ increases, $R$ must decrease. Therefore, the mass shift to the right of $1 \mathrm{TeV}$ indicates the decreasing of compactification radius.

3.3. The Production Cross Section of Graviton Emission Multiplied by Branching Ratio in RS Model. As is known, the discovery of a Higgs boson, $h$, at the LHC motivates the searches for physics beyond SM in channels involving Higgs boson. Higgs pair production is predicted by RS model with KK graviton, $G_{\mathrm{KK}}$, emission that may decay to a pair of Higgs bosons. Such experimental searches were performed by ATLAS Collaboration, [20] at $13 \mathrm{TeV}$, presented in Figure 7.

From [12], branching fractions of graviton decay to SM particles are taken, presented in Figure 8. The predictions 


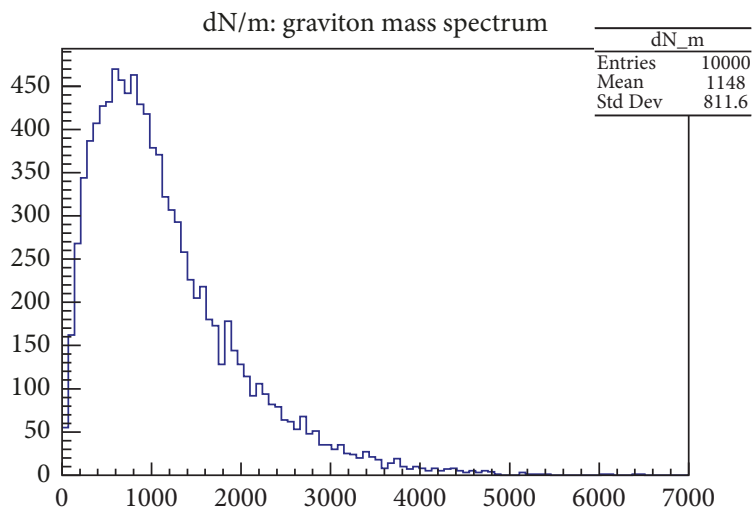

(a)

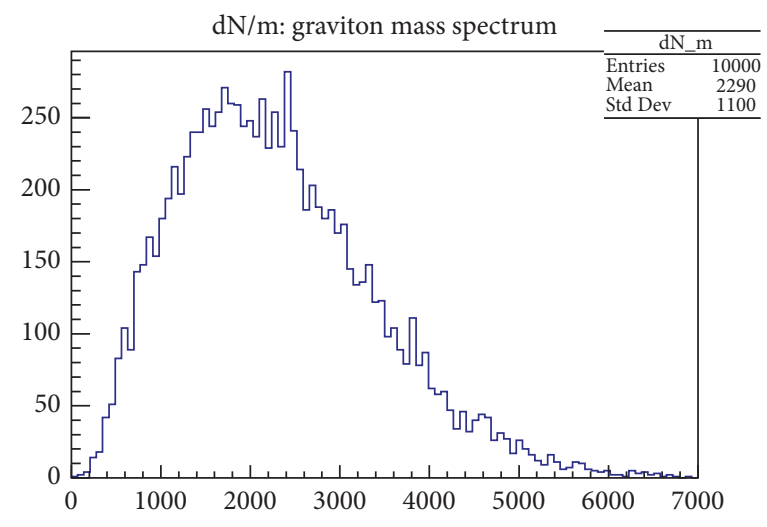

(b)

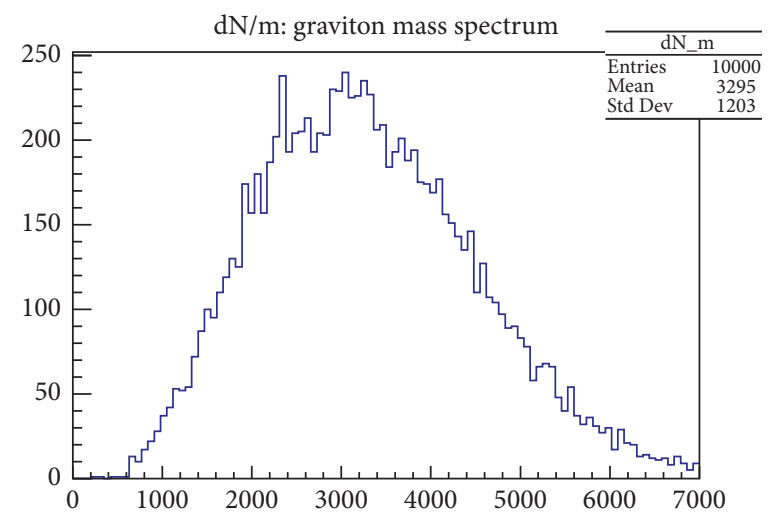

(c)

FIGURE 6: Graviton mass spectrum within monojet LED model for G jet emission process gg $\rightarrow G g$ for (a) $n=2$, (b) $n=4$, and (c) $n=6$ at the center of mass energies of $14 \mathrm{TeV}$.

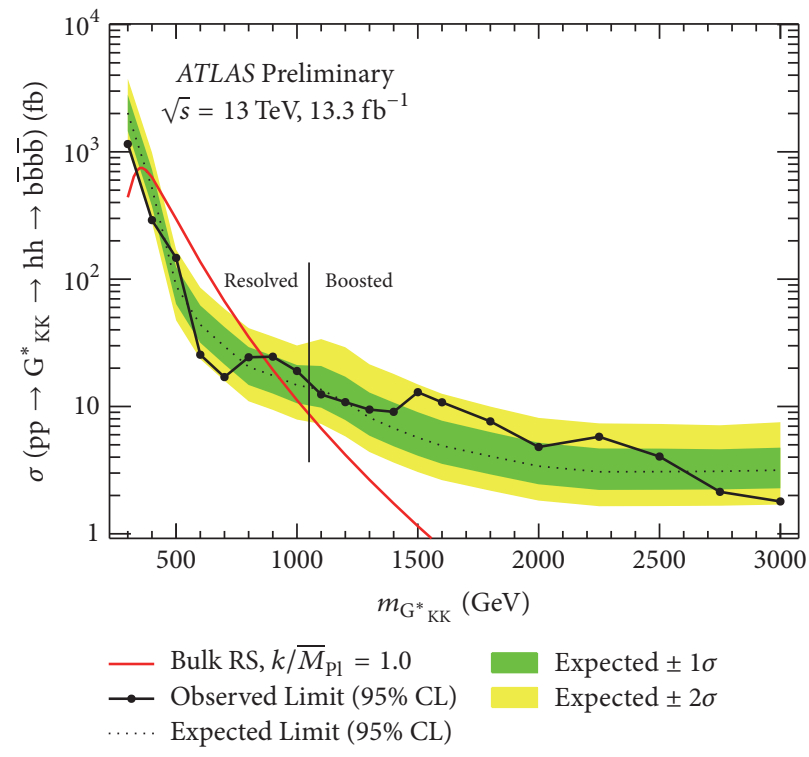

FIGURE 7: The expected and observed upper limit for $\mathrm{pp} \rightarrow G_{\mathrm{KK}} \rightarrow h h \rightarrow b \bar{b} b \bar{b}$ in the bulk RS model with $k / M=1$ at the $95 \%$ confidence level. 


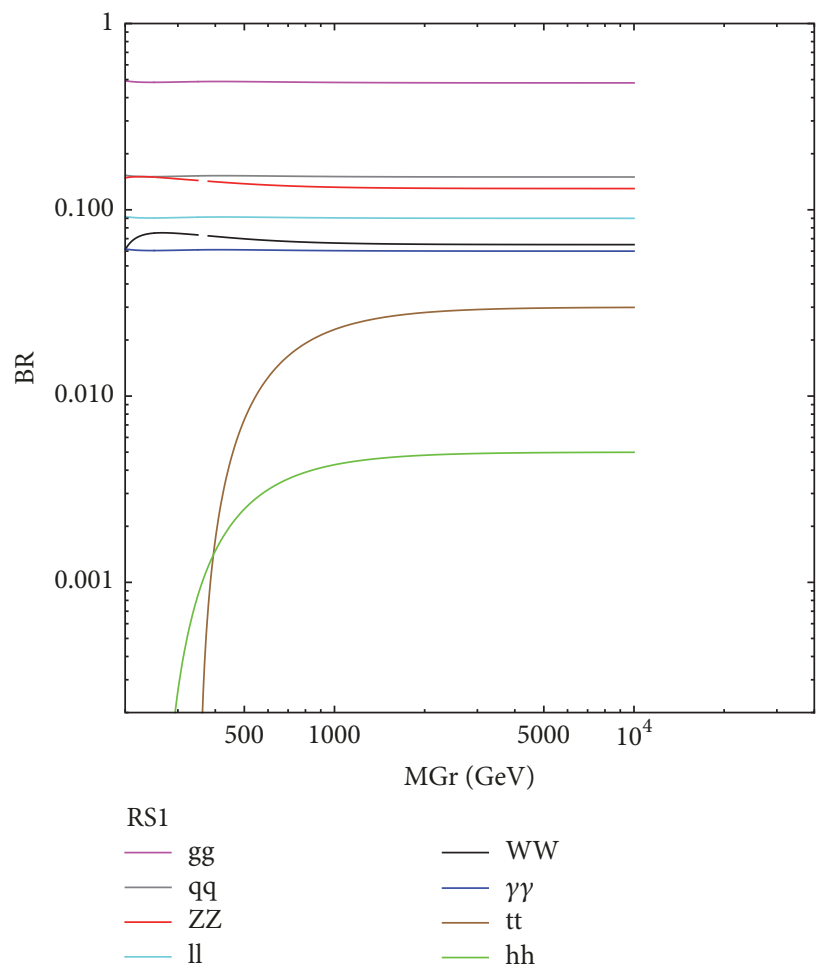

FIGURE 8: Branching fractions of graviton, G (from [12]).

for decay were updated using state-of-the-art computation tools with the highest branching fraction to dijet final states.

We will consider three processes of graviton decay, $\mathrm{G} \rightarrow$ $g g, \mathrm{G} \rightarrow l l$, and $\mathrm{G} \rightarrow h h$, for further $\sigma \times \mathrm{Br}$ calculations within RS scenario for graviton production process gg $\rightarrow$ $G$ at $13 \mathrm{TeV}, 14 \mathrm{TeV}$, and $100 \mathrm{TeV}$ at the center of mass energies. In Figure 9, our calculations performed with the help of Pythia8.2 are presented, with parameters $k / M=1$ and 0.1 .

From Figure 9, we see the dependence of the $\sigma \times \mathrm{Br}$ calculations on the parameter $k / M$ and on the energy at the center of mass. Comparison of Figure $9(a)$ and $9(b)$ shows that the resonance peak shifts from $5 \mathrm{TeV}$ to $7 \mathrm{TeV}$ with increasing of energy at the collider. This mass shift of $2 \mathrm{TeV}$ for case (b) indicates decreasing of the compactification radius of $\mathrm{RS}$ model according to hierarchy formula. In the case of Figure $9(\mathrm{c})$, we can see that there is no peak at $100 \mathrm{TeV}$ and that the cross section for the formation of the resonance as a function of energy is observed to decrease.

\section{Conclusion}

The modern high energy physics is connected with experimental searches of new physics beyond the SM. These searches are connected not only with new possibilities of modern accelerating technics but also with problems of SM physics. The SM problems are not only of theoretical character but also of experimental one, which is confirmed by modern experiments on the Higgs boson. Our work is dedicated to the studying of the properties of the new particles predicted by the theories of extra dimensions. Within three models, $\mathrm{ADD}, \mathrm{RS}$, and $\mathrm{TeV}^{-1}$, we have calculated the production cross sections of massive graviton formation as well as the production of $\mathrm{KK}$ modes of gauge bosons depending on the energies of the modern and future colliders. Within LED model, the behavior of graviton mass spectrum for $\mathrm{G}$ jet emission processes for different numbers of extra dimension ( $n=2,4$, and 6 ) was studied and clear periodicity of peaks shifted by $1 \mathrm{TeV}$ was seen with an increase in the number of extra dimensions by 2 . The graviton mass spectrum for three graviton, $G$, emission processes was also investigated: (a) gg $\rightarrow G g$, (b) qg $\rightarrow G q$, and (c) $q \bar{q} \rightarrow$ $\mathrm{Gg}$ at $14 \mathrm{TeV}$ at the LHC. The experimental searches for KK graviton emission and decay to a pair of Higgs bosons, performed by ATLAS Collaboration at $13 \mathrm{TeV}$, stimulated us to perform calculations at different parameters and energies within RS model. Our calculations of $\sigma \times B r$ shows that the resonance peak shifts from $5 \mathrm{TeV}$ to $7 \mathrm{TeV}$ with increasing of energy at the colliders from $13 \mathrm{TeV}$ to $14 \mathrm{TeV}$ as well as the absence of peak at energy of $100 \mathrm{TeV}$ at the center of mass energies.

\section{Conflicts of Interest}

The authors declare that there are no conflicts of interest regarding the publication of this paper. 


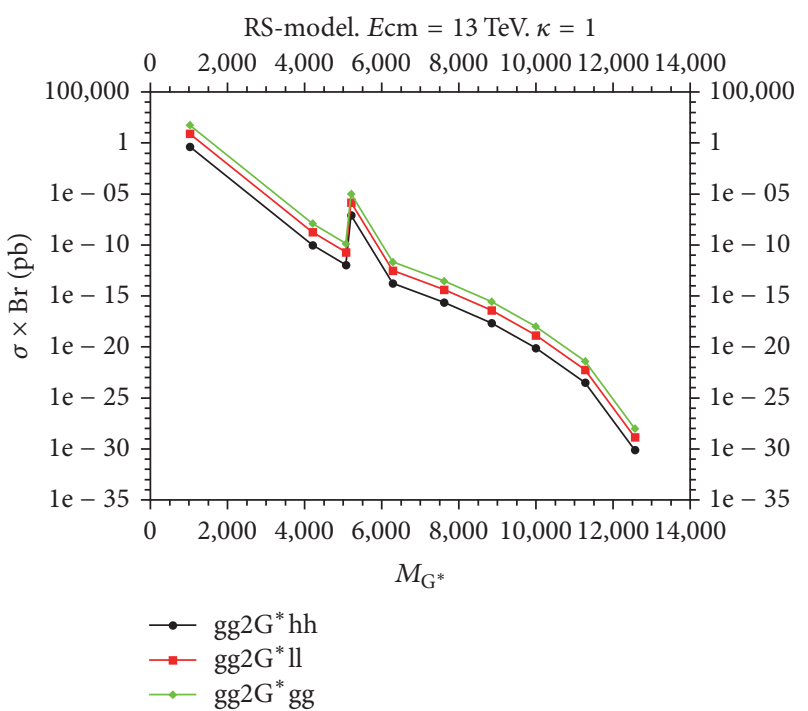

(a)

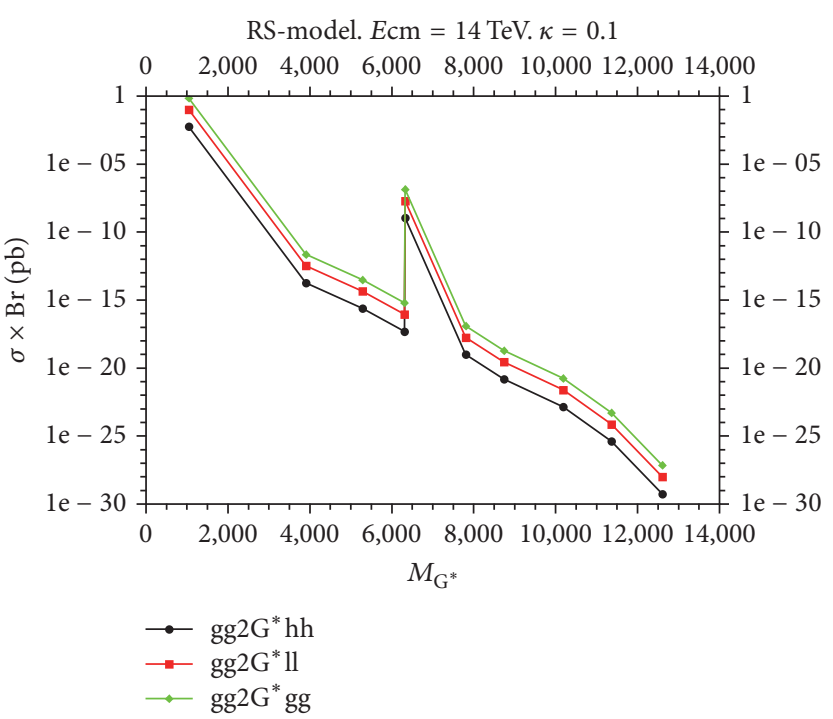

(b)

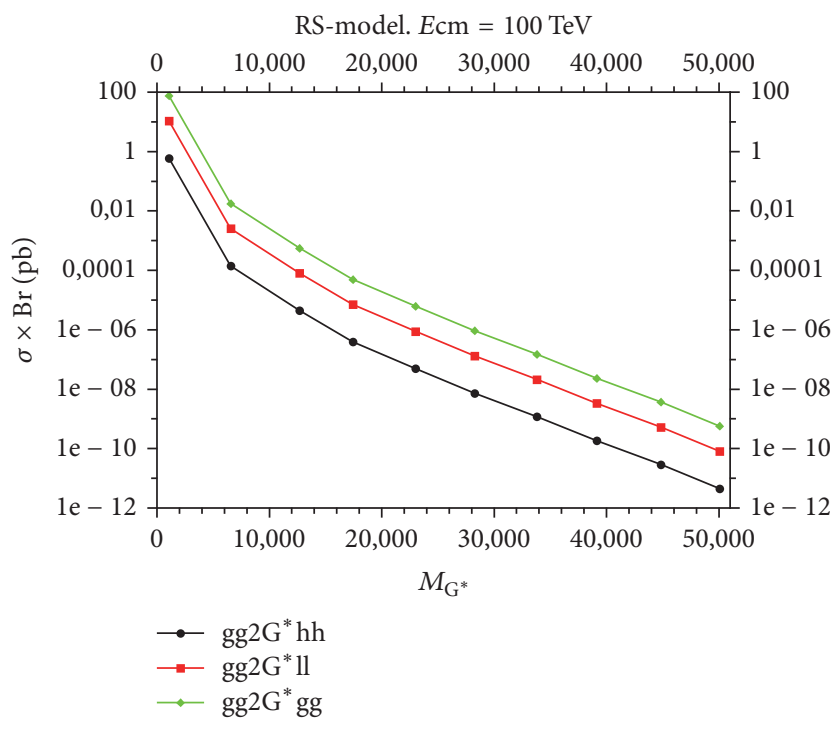

(c)

FiguRE 9: $\sigma \times B r$ for graviton production and decay as the function of graviton mass, $M_{G}$, at (a) $13 \mathrm{TeV}, k / M=1 ;$ (b) $14 \mathrm{TeV}, k / M=0.1 ;$ and (c) $100 \mathrm{TeV}, k / M=0.1$.

\section{References}

[1] Y. A. Kubyshin, Models with Extra Dimensions and Their Phenomenology, 2001.

[2] T. Kaluza, "Zum Unitatsproblem in der Physik," Sitzungsberichte der Koniglich Preußischen Akademie der Wissenschaften (Mathematical Physics), pp. 966-972, 1921.

[3] O. Klein, "Quantentheorie und fünfdimensionale Relativitätstheorie," Zeitschrift für Physik, vol. 37, no. 12, pp. 895-906, 1926.

[4] M. D. Maia, "The Poincare conjecture and the cosmological constant," International Journal of Modern Physics: Conference Series, vol. 03, pp. 195-202, 2011.

[5] N. Arkani-Hamed, S. Dimopoulos, and G. Dvali, "The hierarchy problem and new dimensions at a millimeter," Physics Letters $B$, vol. 429, no. 3-4, pp. 263-272, 1998.
[6] N. Arkani-Hamed, S. Dimopoulos, and G. Dvali, "Phenomenology, astrophysics and cosmology of theories with submillimeter dimensions and TeV scale quantum gravity," Physical Review D, vol. 59, article 086004, 1999.

[7] L. Randall and R. Sundrum, "A large mass hierarchy from a small extra dimension," Physical Review Letters, vol. 83, no. 17, pp. 3370-3373, 1999.

[8] L. Randall and R. Sundrum, "An alternative to compactification," Physical Review Letters, vol. 83, no. 23, pp. 4690-4693, 1999.

[9] S. Maruyama, for the ATLAS, and CMS Collaborations, "Searches for Exotic Phenomena at ATLAS and CMS," High Energy Physics - Experiment, 2014.

[10] I. Antoniadis, "A possible new dimension at a few TeV," Physics Letters B, vol. 246, no. 3-4, pp. 377-384, 1990. 
[11] T. Sjöstrand, S. Ask, J. R. Christiansen et al., "An introduction to PYTHIA 8.2," Computer Physics Communications, vol. 191, no. 1, pp. 159-177, 2015.

[12] A. Carvalho, Gravity Particles from Warped Extra Dimensions, Predictions for LHC, 2017.

[13] P. Hořava and E. Witten, "Heterotic and Type I String Dynamics from Eleven Dimensions," Nuclear Physics B, vol. 460, no. 3, pp. 506-524, 1996.

[14] J. Bijnens, P. Eerola, M. Maul, A. Månsson, and T. Sjöstrand, "QCD signatures of narrow graviton resonances in hadron colliders," Physics Letters B, vol. 503, no. 3-4, pp. 341-348, 2001.

[15] The ATLAS Collaboration, "Search for diboson resonances with boson-tagged jets in $p p$ collisions at $s=13 \mathrm{TeV}$ with the ATLAS detector," Physics Letters B, vol. 777, no. 91, 2017.

[16] S. Ask, I. V. Akin, L. Benucci, A. De Roeck, M. Goebel, and J. Haller, "Real emission and virtual exchange of gravitons and unparticles in Pythia8," Computer Physics Communications, vol. 181, no. 9, pp. 1593-1604, 2010.

[17] G. Azuelos, P.-H. Beauchemin, and C. P. Burgess, "Phenomenological constraints on extra-dimensional scalars," Journal of Physics G: Nuclear and Particle Physics, vol. 31, pp. 1-20, 2005.

[18] G. Bella, E. Etzion, N. Hod, and M. Sutton, "Introduction to the MCnet Moses project and Heavy gauge bosons search at the LHC," High Energy Physics - Experiment, 2010.

[19] G. Bella, E. Etzion, N. Hod, Y. Oz, Y. Silver, and M. Sutton, "A search for heavy Kaluza-Klein electroweak gauge bosons at the LHC," Journal of High Energy Physics, vol. 2010, no. 9, article no. 025, 2010.

[20] The ATLAS Collaboration, "Search for pair production of Higgs bosons in the $b b b b$ final state using proton-proton collisions at $s=13 \mathrm{TeV}$ with the ATLAS detector," ATLAS-CONF-049, 2016. 

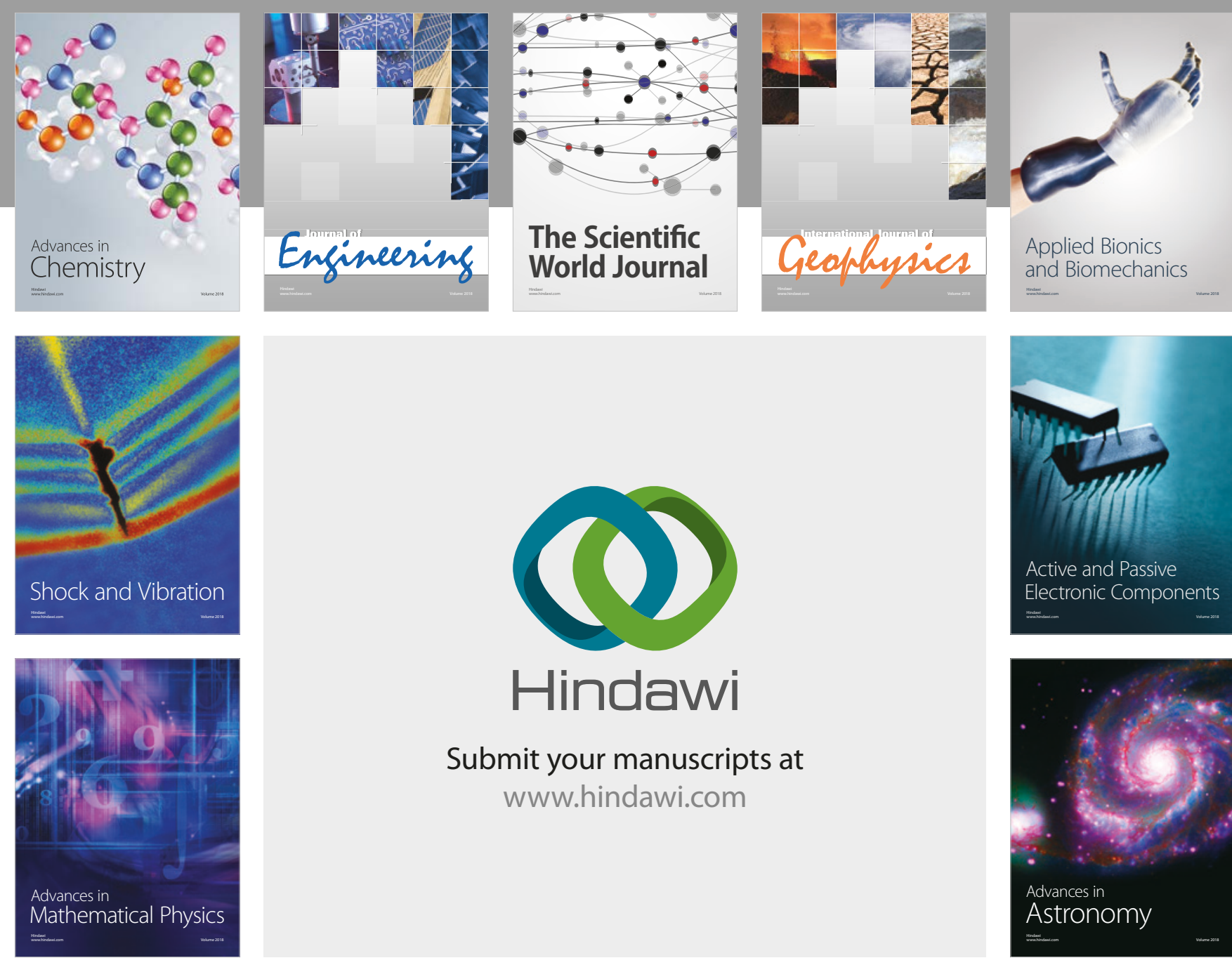

Submit your manuscripts at

www.hindawi.com

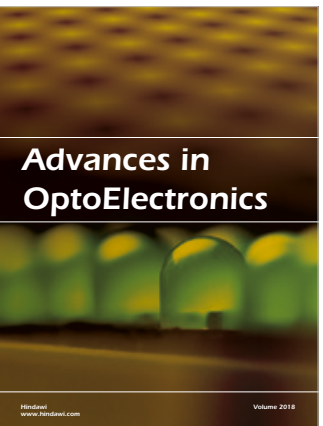

\section{Rotcting Machinery}
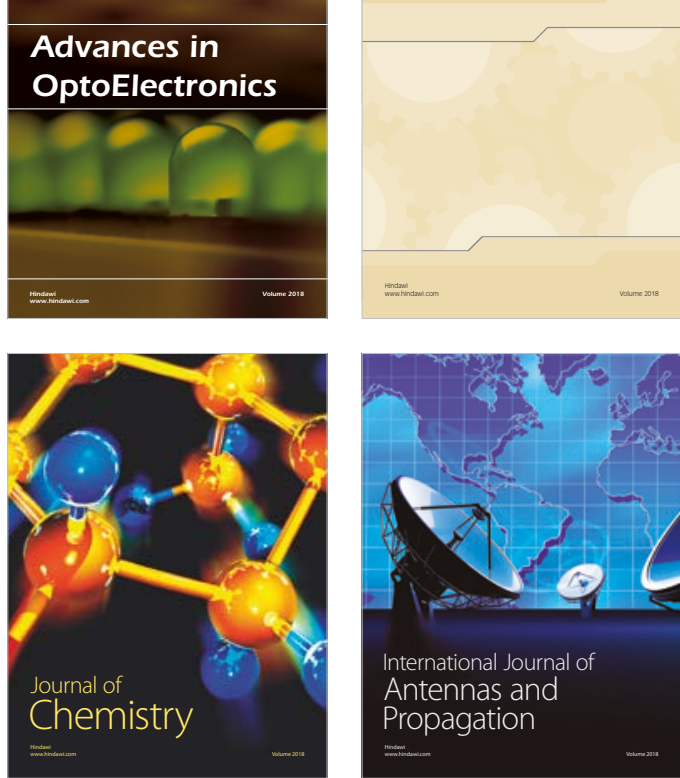

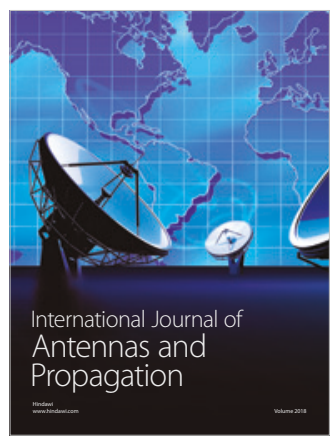

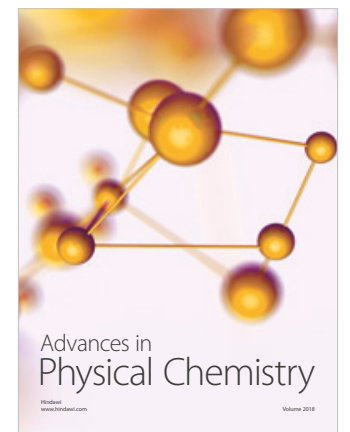

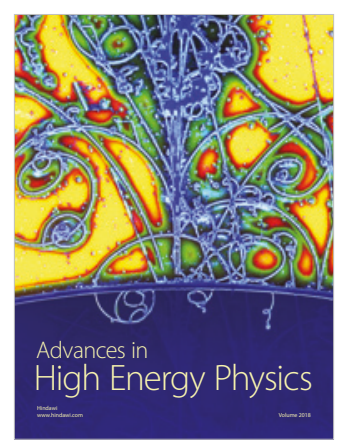

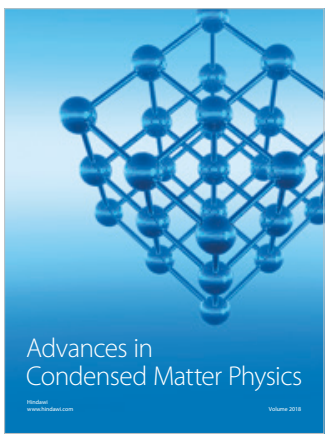

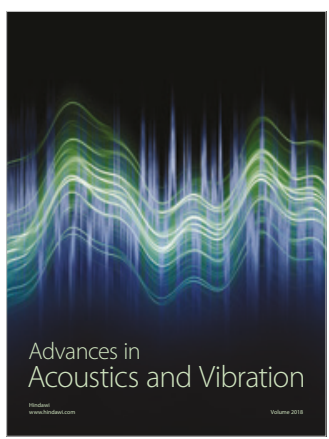

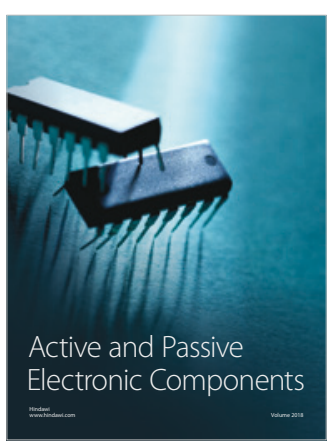
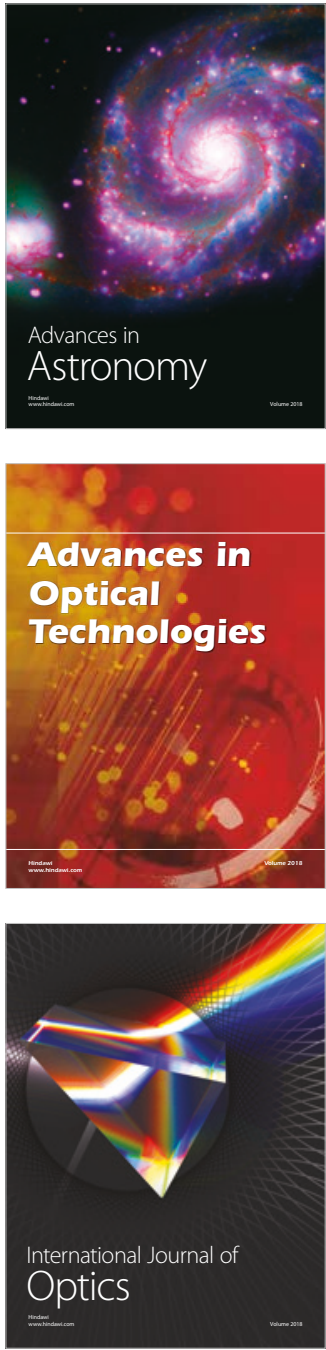